\title{
Stalled Decline in Fertility in Ecuador
}

Many developing countries have experienced rapid declines in fertility since the 1960s. Although some have already reached the replacement level of 2.1 lifetime births per woman, and others are expected to do so in the future, the decrease in fertility in other countries has slowed or even stalled. ${ }^{1}$ Ecuador clearly falls into the latter category. After remaining at approximately 7.0 lifetime births per woman between 1955 and 1969, its total fertility rate (TFR) steadily declined throughout the 1970s and 1980s, reaching 3.8 births in 1989; the average yearly decline over this period was 0.17 births per year. However, the decline slowed between 1989 and 2004 to an average of 0.03 births per year, and the country's TFR in 2004 was 3.3 births per woman.

Other demographic and socioeconomic trends are consistent with the slowing of Ecuador's fertility decline between 1989 and 2004. The decline in child mortality-one of the prerequisites for a sustained demographic transition ${ }^{2}-$ similarly slowed: Mortality fell from 82 deaths per 1,000 children younger than five in 1987 to 39 deaths in 2000 , and then to 35 deaths by $2004 .^{3}$ The major economic crisis that occurred in 1999 may have further slowed the fertility decline by reducing the number of jobs and thus the opportunity cost for women of childbearing and child rearing, which had increased during the preceding period of economic development. ${ }^{4,5}$

The availability of modern contraceptives plays a key role in fertility decline. In a recent study, a leveling off in contraceptive use was accompanied by stalled fertility in several less-developed countries. ${ }^{1}$ Donor funding for family planning programs in Ecuador declined during the 1990s, leading to increased user fees for contraceptives. ${ }^{6}$ Populationbased survey data show that despite the increase in fees, overall contraceptive prevalence increased at a constant rate between 1989 and 2004. ${ }^{3}$ Nonetheless, the substantial increase in contraceptive use did not result in further fertility decline in Ecuador, a unique exception in the literature. This comment considers proximate determinants of fertility (union status, contraceptive use and postpartum infecundability) for the 1994-2004 period, and examines whether the patterns of these determinants and of fertility desire are consistent with the stalled fertility decline in Ecuador. We pay close attention to differences according to women's geographic and educational characteristics.

This comment uses data from the Ecuador Demographic, Maternal and Infant Health Surveys conducted in 1994, 1999 and 2004 by the Center for Studies of Population and Social Development, with technical assistance from the U.S. Centers for Disease Control and Prevention. 3,7,8 The samples consist of 13,582 women aged 15-49 in the 1994 survey, 14,285 in 1999 and 10,814 in 2004. These samples are representative of populations in 17 geographic units in Ecuador-10 provinces in the Sierra region, five in the Costa region, and the entire Insular (Galapagos Islands) and Amazonia regions.

The mountainous Sierra region includes the country's capital, Quito. The Costa, which borders the Pacific Ocean, includes the main commercial port city, Guayaquil. The national population is mainly mestiza (mixed race); according to the most recent survey, Amazonia is the region with the largest proportion of indigenous people (17\%), followed by the Sierra (11\%) and the Costa (1\%). The Insular and Amazonia regions were not included in the 1994 survey, so the national estimates for 1994 are not entirely comparable with those for 1999 and 2004. However, because the sensitivity analysis suggests that estimates with these two regions do not differ substantially from those without them, estimates for all regions are presented.

The consistency and quality of fertility measurements in these three surveys are assessed by comparing cohortspecific fertility rates for each survey period on the basis of retrospective five-year birth histories; estimates are available for the Sierra and Costa regions separately, and for the country as a whole. The results indicate a high degree of similarity among these estimates.

\section{Fertility Rates by Subgroup}

The national TFR in Ecuador was 3.6 lifetime births per woman in 1994, 3.4 births in 1999 and 3.3 births in 2004 (Table 1, page 204). The 95\% confidence intervals for these TFRs overlap substantially, particularly for the two most recent surveys, and t tests indicate that the slight differences between any two successive estimates are not statistically significant. Hence, it appears that fertility decline has stabilized in Ecuador over the last decade. In the second half of the 20th century, the decline in fertility in the Costa region was more rapid than that in the Sierra region. ${ }^{9}$ However, the Costa region did not experience a steady decrease in TFR in the last decade, while the Sierra region experienced a significant decline from 3.9 to 3.1 births per woman between the 1994 and 2004 surveys. Furthermore, much of the decline in the Sierra came from the rural areas, where the TFR dropped from 4.8 to 3.7 births. As a result, the fertility rates in the Costa and Sierra regions were significantly different in 1994, but not in 2004.

Regarding women's educational attainment over the

\section{By Kanako Ishida, Paul Stupp and Jose Ordoñez Sotomayor}

Kanako Ishida is Oak Ridge Institute for Science and Education Fellow, and Paul Stupp is a statistician, both in the Division of Reproductive Health, Centers for Disease Control and Prevention, Atlanta, GA, USA. Jose Ordoñez Sotomayor is director, Center for Studies of Population and Social Development, Quito, Ecuador. 
TABLE 1. Total fertility rates (and $95 \%$ confidence intervals) among women aged 15-49, by regional and educational subgroup, according to survey year

\begin{tabular}{llll} 
Subgroup & 1994 & 1999 & 2004 \\
\hline All & $\mathbf{3 . 6 1 ( 3 . 4 6 - 3 . 7 7 )}$ & $\mathbf{3 . 3 8 ( 3 . 2 4 - 3 . 5 2 )}$ & $\mathbf{3 . 2 7}(\mathbf{3 . 1 5 - 3 . 3 9 )}$ \\
$\begin{array}{l}\text { Region } \\
\text { Sierra }\end{array}$ & & & \\
$\quad$ Quito & $3.91(3.69-4.13)$ & $3.59(3.39-3.79)$ & $3.14(2.97-3.31)$ \\
$\quad$ Other urban areas & $3.01(2.68-3.34)$ & $2.76(2.46-3.07)$ & $2.73(2.36-3.10)$ \\
$\quad$ Rural areas & $2.95(2.71-3.20)$ & $2.90(2.66-3.13)$ & $2.58(2.33-2.82)$ \\
Costa & $4.83(4.51-5.16)$ & $4.42(4.11-4.74)$ & $3.68(3.43-3.94)$ \\
$\quad$ Guayaquil & $3.33(3.12-3.54)$ & $3.01(2.83-3.20)$ & $3.32(3.14-3.50)$ \\
$\quad$ Other urban areas & $2.67(2.35-3.00)$ & $2.48(2.17-2.80)$ & $2.97(2.65-3.28)$ \\
$\quad$ Rural areas & $3.19(2.91-3.47)$ & $2.92(2.69-3.14)$ & $3.13(2.87-3.40)$ \\
Amazonia & $4.20(3.77-4.63)$ & $3.94(3.55-4.34)$ & $4.04(3.71-4.38)$ \\
Insular & & & \\
& $\mathrm{u}$ & $5.49(4.79-6.19)$ & $4.19(3.50-4.88)$ \\
Yrs. of education & $\mathrm{u}$ & $2.27(1.74-2.79)$ & $2.74(1.95-3.52)$ \\
$0-5$ & & & \\
6 & $5.66(5.30-6.01)$ & $5.29(4.96-5.63)$ & $4.93(4.58-5.27)$ \\
$7-11$ & $3.96(3.73-4.18)$ & $3.84(3.64-4.04)$ & $3.97(3.76-4.19)$ \\
$\geq 12$ & $3.07(2.87-3.26)$ & $3.26(3.04-3.49)$ & $3.03(2.83-3.23)$ \\
\hline
\end{tabular}

Notes: The 1994 estimate is based on women's birth reports for the period June 1989-May 1994, the 1999 estimate is based on reports for March 1994-February 1999 and the 2004 estimate is based on reports for July 1999-June 2004. All estimates are weighted. u=unavailable, because these regions were not sampled in 1994.

decade, the only significant decline in TFR was observed for those who had 0-5 years of schooling (from 5.7 births in 1994 to 4.9 births in 2004). Women with more education showed virtually no decline at any level of schooling over that period: The fertility level stabilized at 4.0 births for women with six years of schooling, at 3.0 births for those with 7-11 years of schooling and at 2.4 births for those with 12 or more years of schooling. Thus, the fertility gap between women with the lowest and the highest levels of educational attainment narrowed in the 2004 survey.

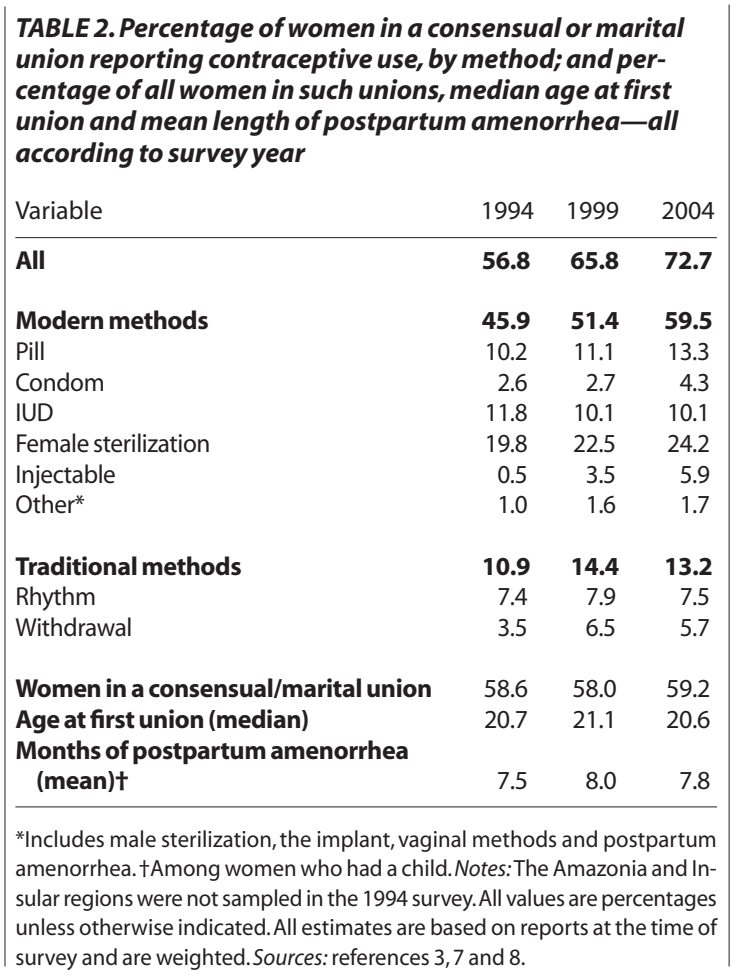

\section{Proximate Determinants of Fertility}

We used the Bongaarts model ${ }^{10-12}$ to assess whether levels of the proximate determinants of fertility changed over the three survey periods, and whether any such changes might explain the stabilization of fertility in Ecuador. Between 1994 and 2004, virtually no change was observed in the proportion of women in a consensual or marital union (average, 59\%), in the median age at first union (average, 21) or in the mean length of postpartum amenorrhea (average, eight months; Table 2). However, use of contraceptive methods (modern and traditional combined) increased from $57 \%$ in 1994 to $73 \%$ in 2004 . The largest increases were for the pill, the injectable and female sterilization, which together constituted $81 \%$ of the increase in contraceptive use (not shown).

In the Bongaarts model, the relative importance of the proximate determinants of fertility (after conversion to indices) can vary from 0 to 1 , where 1 indicates no inhibition of fertility by the determinant and values closer to 0 indicate increasing inhibition (Table 3). The product of these indices and the hypothetical maximum potential fertilityestimated to be 15.3 lifetime births per woman-yields the predicted TFR. The large increase in the proportion of women who used contraceptives translates into the large decrease in the value for contraception, which fell from 0.42 in 1994 to 0.26 in 2004. The importance of this decrease is illustrated by the decline in the predicted TFR, from 2.92 to 1.81 births per woman. However, the growing gap between the predicted and the observed TFRs-from -0.69 in 1994 to -1.46 in 2004-suggests that the use of contraceptives did not have the expected effect of inhibiting fertility, as the TFR was essentially unaffected by the overall increase in contraceptive use over the last decade.

The lack of association between contraceptive prevalence rate (CPR) and region or education level is evident in the Costa region: Virtually no change in fertility was observed between 1994 and 2004, but the CPR increased at a similar rate in the Costa and Sierra regions (Table 4). In the Sierra region, however, where the fertility level dropped significantly over the decade, a negative association between CPR and TFR was seen, as expected. In particular, the fertility-reducing effect of rising contraceptive use in rural areas of the Sierra is clearly evident. Contraceptive use also increased over the decade for women at all educational levels. However, for women with six or more years of schooling-whose TFR was already lower than those for women with less schooling-fertility was not responsive to the increases in contraceptive prevalence between the surveys.

\section{Fertility Preferences}

At each survey, respondents were asked to classify all births in the survey period as wanted, mistimed or unwanted. Among 15-19-year-olds, the proportion of mistimed births increased over the decade (from 13\% to $23 \%$ ), while the proportion of unwanted births changed little (from $8 \%$ to $9 \%$; Table 5). In the other age-groups, 


\begin{tabular}{|lccc|}
\multicolumn{4}{|l}{ TABLE 3. Indices of proximate determinants of fertility, and } \\
selected fertility measures, by survey year \\
Variable & 1994 & 1999 & \\
\hline Proportion of women in union & 0.586 & 0.580 & 0.592 \\
Contraception & 0.424 & 0.336 & 0.263 \\
$\quad$ Proportion using contraceptives & 0.568 & 0.658 & 0.727 \\
$\quad$ Average use-effectiveness & 0.938 & 0.933 & 0.938 \\
Postpartum infecundability & 0.769 & 0.755 & 0.760 \\
Maximum potential fertility & 15.3 & 15.3 & 15.3 \\
Predicted TFR & 2.92 & 2.25 & 1.81 \\
Observed TFR & 3.61 & 3.38 & 3.27 \\
Difference in TFRs & & & \\
$\quad$ (predicted-observed) & -0.69 & -1.13 & -1.46 \\
\hline
\end{tabular}

Notes: The Amazonia and Insular regions were not sampled in the 1994 survey. All estimates are weighted. Proportion of women in union $=\mathrm{C}_{\mathrm{m}^{\prime}}$; contraception $=C_{c^{\prime}}$ proportion using contraceptives $=u$; average use-effectiveness $=$ e; postpartum infecundability $=\mathrm{C}_{\mathrm{i}} ; \mathrm{i}=$ months of postpartum infecundability; total fertility rate=TFR. $C_{c}=1-1.08^{*} u^{*} e . C_{i}=20 /(18.5+i)$. Predicted TFR $=C_{m}{ }^{*} C_{c}{ }^{*} C_{i}^{*} 15.3$.

the proportions of wanted and unwanted births did not change substantially. In addition, the mean of the ideal number of children desired by Ecuadorean women remained level: 2.7 in 1994, 2.5 in 1999 and 2.6 in 2004 (not shown). The striking absence of change in this preference is consistent with the lack of decline in fertility over the decade, and may explain why TFRs did not decrease with increased contraceptive use: Women were reaching their desired fertility level, and hence their contraceptive use was directed at spacing their births, not at reducing the total number of children. Furthermore, the gap between the observed TFR and the mean ideal number of children across subregions may have been too small to produce a substantial decrease between 1994 and 2004.

\section{Conclusions}

The decline in fertility has leveled off in Ecuador at about three lifetime births per woman. This stabilization was particularly evident in the Costa region, where the fertility rate was already low in 1994; regional differences in fertility levels that existed in the last half of the 20th century have now been largely erased. Women with at least six years of schooling did not experience a significant decline in fertility, and rates have stabilized among women at all education levels except $0-5$ years of schooling. The smaller but still significant differences in TFR by education level suggest that an increase in educational attainment among
TABLE 4. Contraceptive prevalence rates (and 95\% confidence intervals) by regional and educational subgroup, according to survey year

\begin{tabular}{llll} 
Subgroup & 1994 & 1999 & 2004 \\
\hline All & $\mathbf{5 6 . 8 ( 5 4 . 9 - 5 8 . 7 )}$ & $\mathbf{6 5 . 8}(\mathbf{6 4 . 3}-\mathbf{6 7 . 2})$ & $\mathbf{7 2 . 7}(\mathbf{7 1 . 3 - 7 4 . 1})$ \\
Region & & & \\
Sierra & & & \\
$\quad$ Quito & $55.2(52.5-57.8)$ & $64.1(61.7-66.4)$ & $70.5(68.3-72.6)$ \\
$\quad$ Other urban areas & $70.1(65.6-74.4)$ & $74.4(70.5-77.9)$ & $78.1(74.0-81.7)$ \\
$\quad$ Rural areas & $68.0(64.7-71.0)$ & $71.3(67.7-74.6)$ & $76.5(73.4-79.4)$ \\
Costa & $42.1(38.3-46.0)$ & $55.2(51.4-59.0)$ & $63.7(60.3-67.1)$ \\
$\quad$ Guayaquil & $58.2(55.6-60.8)$ & $68.4(66.5-70.3)$ & $75.6(73.5-77.5)$ \\
$\quad$ Other urban areas & $65.6(61.1-70.0)$ & $70.3(67.1-73.3)$ & $77.1(72.9-80.8)$ \\
$\quad$ Rural areas & $62.5(59.3-65.7)$ & $70.3(67.7-72.7)$ & $75.4(72.3-78.3)$ \\
Amazonia & $47.3(42.7-52.0)$ & $63.7(59.4-67.8)$ & $74.1(70.7-77.2)$ \\
Insular & & & \\
Yrs. of education & $\mathrm{u}$ & $53.9(45.8-61.7)$ & $65.3(57.8-72.2)$ \\
$0-5$ & $\mathrm{u}$ & $76.2(69.2-81.9)$ & $76.5(67.7-83.5)$ \\
6 & & & \\
7-11 & $37.5(34.2-41.0)$ & $52.1(48.7-55.5)$ & $61.8(58.4-65.0)$ \\
$\geq 12$ & $54.9(52.4-57.4)$ & $62.8(60.5-65.1)$ & $69.9(67.4-72.3)$ \\
\hline & $63.6(60.6-66.4)$ & $67.4(64.9-69.9)$ & $76.9(74.2-79.4)$ \\
& $71.7(69.1-74.1)$ & $76.5(74.6-78.3)$ & $79.1(76.9-81.3)$ \\
\hline
\end{tabular}

Notes: All estimates are weighted. u=unavailable, because these regions were not sampled in the 1994 survey.

Ecuadoreans may facilitate a future decline in fertility, ${ }^{13}$ but the process will be slow.

Ecuador's situation is unique among developing countries, not only in these small differences in fertility by geographic and educational characteristics, but in showing no association between trends in fertility and contraceptive use. The latter increased from 57\% to 73\% between 1994 and 2004, and was expected, according to the Bongaarts model, to translate to a reduction of one lifetime birth per woman. However, instead of continuing its decline, total fertility stabilized, particularly among women living in the Costa region and among those with at least six years of schooling. Finally, the lack of change in the level of unwanted births and the desired number of children across the same period is consistent with the stalled decline in fertility, and the increased use of contraceptives likely indicates that women are focusing on the timing of their births, albeit with limited success.

The absence of a strong association between fertility rates and contraceptive use, and the small gap between the observed TFR and the mean ideal number of children reported in 2004, suggest that prospects for further fertility decline in Ecuador are limited in the near future. Indeed,

\begin{tabular}{|c|c|c|c|c|c|c|c|c|c|}
\hline \multirow[t]{2}{*}{ Age-group } & \multicolumn{3}{|l|}{1994} & \multicolumn{3}{|l|}{1999} & \multicolumn{3}{|l|}{2004} \\
\hline & Wanted & Mistimed & Unwanted & Wanted & Mistimed & Unwanted & Wanted & Mistimed & Unwanted \\
\hline $15-19$ & 79.5 & 12.6 & 8.0 & 72.5 & 19.3 & 8.3 & 68.4 & 23.1 & 8.5 \\
\hline $20-24$ & 71.3 & 17.1 & 11.6 & 64.4 & 20.7 & 15.0 & 64.5 & 20.6 & 14.9 \\
\hline $25-29$ & 67.7 & 16.2 & 16.1 & 59.9 & 18.8 & 21.4 & 64.8 & 18.1 & 17.2 \\
\hline $30-34$ & 63.2 & 12.9 & 23.9 & 57.2 & 13.7 & 29.1 & 62.2 & 13.2 & 24.6 \\
\hline $35-39$ & 59.5 & 8.0 & 32.5 & 54.2 & 7.3 & 38.6 & 58.0 & 9.7 & 32.4 \\
\hline $40-44$ & 51.1 & 4.7 & 44.2 & 46.7 & 4.8 & 48.5 & 50.1 & 2.4 & 47.4 \\
\hline $45-49$ & 44.8 & 4.7 & 50.5 & 53.3 & 8.9 & 37.9 & 53.9 & 0.0 & 46.1 \\
\hline Total & 68.9 & 14.2 & 17.0 & 62.1 & 17.0 & 20.9 & 63.9 & 17.7 & 18.4 \\
\hline
\end{tabular}


a significant decrease in Ecuadoreans' desired family sizerather than a continuing increase in contraceptive use-is likely needed before the country will experience a substantial decrease in the current fertility rate.

\section{REFERENCES}

1. Bongaarts J, The causes of stalling fertility transitions, Studies in Family Planning, 2006, 37(1):1-16.

2. Coale AJ, Population growth and economic development: the case of Mexico, Foreign Affairs, 1978, 56(2):415-429.

3. Center for Studies of Population and Social Development (CEPAR) et al., Ecuador Encuesta Demográfica y de Salud Materna e Infantil (ENDEMAIN), 2004: Informe Final, Quito, Ecuador: CEPAR, 2005.

4. Lucero JA, Crisis and contention in Ecuador, Journal of Democracy, 2001, 12(2):59-73.

5. Falconí-Benítez F, Integrated assessment of the recent economic history of Ecuador, Population and Environment, 2001, 22(3):257-279.

6. Bratt JH et al., The impact of price changes on demand for family planning and reproductive health services in Ecuador, Health Policy Planning, 2002, 17(3):281-287.

7. CEPAR et al., Ecuador Encuesta Demográfica y de Salud Materna e Infantil (ENDEMAIN), 1994: Informe Final, Quito, Ecuador: CEPAR, 1995.

8. CEPAR et al., Ecuador Encuesta Demográfica y de Salud Materna e Infantil (ENDEMAIN), 1999: Informe Final, Quito, Ecuador: CEPAR, 2001.

9. CEPAR, Transicion de la Fecundidad en el Ecuador, Quito, Ecuador: CEPAR, 1991.

10. Bongaarts J, A framework for analyzing the proximate determinants of fertility, Population and Development Review, 1978, 4(1): 105-132.

11. Bongaarts J, The fertility-inhibiting effects of the intermediate fertility variables, Studies in Family Planning, 1982, 13(6/7):179-189.

12. Bongaarts J and Potter RG, Fertility, Biology, and Behavior, New York: Academic Press, 1983.

13. Bongaarts J, Completing the fertility transition in the developing world: the role of educational differences and fertility preferences, Population Studies, 2003, 57(3):321-335.

Author contact:kanakoi@ucla.edu 